\title{
A new discrete Hardy-type inequality with kernels and monotone functions
}

\author{
Aigerim Kalybay' ${ }^{1}$ Lars-Erik Persson ${ }^{2,3^{*}}$ and Ainur Temirkhanova ${ }^{4}$
}

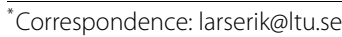
${ }^{2}$ Luleå University of Technology, Luleå, 97187 , Sweden

${ }^{3}$ Narvik University College, P.O. Box 385, Narvik, 8505, Norway Full list of author information is available at the end of the article

\begin{abstract}
A new discrete Hardy-type inequality with kernels and monotone functions is proved for the case $1<q<p<\infty$. This result is discussed in a general framework and some applications related to Hölder's summation method are pointed out.
\end{abstract}

MSC: Primary 26D10; 26D15; secondary 39B82

Keywords: inequality; Hardy-type inequality; kernel; matrix operator; monotone sequence; Oinarov condition

\section{Introduction}

Hardy's famous inequality reads

$$
\int_{0}^{\infty}\left(\frac{1}{x} \int_{0}^{x} f(t) d t\right)^{p} d x \leq\left(\frac{p}{p-1}\right)^{p} \int_{0}^{\infty} f^{p}(x) d x, \quad p>1,
$$

where $f(x)$ is a $p$-integrable non-negative function on $(0, \infty)$. The constant $\left(\frac{p}{p-1}\right)^{p}$ is sharp. Hardy stated his inequality in 1920 (see [1]) and finally proved it in 1925 (see [2]). Since then there has been an enormous development of this inequality to what today is called Hardy-type inequalities. One important reason is that this theory is very important for various applications both in mathematics and the technical sciences. See e.g. [3-5] and the references given there. Let us just mention some important steps in this development. One central problem was to characterize the weights $u(x)$ and $v(x)$ so that the more general Hardy-type inequality

$$
\left(\int_{0}^{\infty}\left(\int_{0}^{x} f(t) d t\right)^{q} u(x) d x\right)^{\frac{1}{q}} \leq C\left(\int_{0}^{\infty} f^{p}(x) v(x) d x\right)^{\frac{1}{p}}
$$

holds for various parameters $p$ and $q$, and to also have some good estimates of the best constant $C$ (the operator norm). Here and in the sequel $C$ does not depend on $f$. This problem is nowadays solved for most cases (see e.g. the books mentioned above). However, for various applications it is also important to consider the mapping properties of the more general operator $H_{k}(f)(x):=\int_{0}^{x} k(x, t) f(t) d t$, where $k(x, t)$ is a kernel (a non-negative and measurable function on $\{(x, t): 0 \leq x<\infty, 0<t \leq x\})$. In applications $k=k(x, t)$ is sometimes called the Green-kernel or unit impulse answer when solving technical problems modeled by linear differential equations. In the case with weighted Lebesgue spaces

(c) 2015 Kalybay et al. This article is distributed under the terms of the Creative Commons Attribution 4.0 International License (http://creativecommons.org/licenses/by/4.0/), which permits unrestricted use, distribution, and reproduction in any medium, provided you give appropriate credit to the original author(s) and the source, provide a link to the Creative Commons license, and indicate if changes were made. 
this problem is equivalent to study the inequality (2) with $H_{1}$ replaced by $H_{k}$. It is still a well-known open problem to characterize (2) with $H_{1}$ replaced by $H_{k}$ without restrictions on $k$. However, the problem is more or less solved for some special cases, e.g. the following:

(a) $k(x, t) \equiv 1$ (the classical case; see e.g. [3, 4] and [5]);

(b) $k(x, t)$ is of product type (see e.g. [5] and the references therein);

(c) $k(x, t)$ satisfies the so-called Oinarov condition (see e.g. [3], Chapter 2, especially p.77);

(d) $k(x, t)$ satisfies some generalized Oinarov condition (see [6] and [7]).

It is also of great interest to investigate all the problems above when the inequalities are studied only on the cone of non-decreasing functions. In particular, such inequalities give precise results concerning embeddings between weighted Lorentz spaces described by different (quasi-)norms. Here, we mention the fundamental paper [8] from 1990 by Sawyer, where he proved his famous 'Sawyer duality principle' for reduction of an inequality for monotone functions to the corresponding inequality for non-negative functions.

There has been a similar development for Hardy-type inequalities in the discrete case. For clarity and as an introduction of the main result of this paper we briefly describe this development in Section 2, where we also compare with the continuous case and formulate our main result. This main result is proved in Section 4. In order to prepare for this proof we state some auxiliary results in Section 3. Finally, we give some applications connected to Hölder's summation method (see [9]) in Section 5.

\section{The discrete case - formulation of the main result}

The discrete form of Hardy's inequality reads

$$
\sum_{n=1}^{\infty}\left(\frac{1}{n} \sum_{k=1}^{n} f_{k}\right)^{p} \leq\left(\frac{p}{p-1}\right)^{p} \sum_{n=1}^{\infty} f_{n}^{p}, \quad p>1,
$$

where $f=\left\{f_{k}\right\}$ is any sequence of non-negative numbers. The constant $\left(\frac{p}{p-1}\right)^{p}$ is sharp also in this case. By applying (1) with step-functions we obtain (3). The discrete inequality corresponding to (2) reads

$$
\left(\sum_{n=1}^{\infty}\left(\sum_{k=1}^{n} f_{k}\right)^{q} u_{n}\right)^{\frac{1}{q}} \leq C\left(\sum_{n=1}^{\infty} f_{n}^{p} v_{n}\right)^{\frac{1}{p}},
$$

where $u=\left\{u_{n}\right\}$ and $v=\left\{v_{n}\right\}$ are sequences of non-negative numbers. Also in this case the characterization of (4) is known for almost all reasonable parameters $p$ and $q$ (see e.g. [4] and the references therein). However, in this case the result does not follow automatically from the corresponding result in the continuous case but must be proved separately. Also in this case it is important to study the more general case when the operator $H(f)(n)=\sum_{k=1}^{n} f_{k}$ is replaced by a more general discrete kernel (matrix) operator $H_{w}(f)(n)=\sum_{k=1}^{n} w_{n, k} f_{k}$, where $w_{n, k}, 1 \leq n \leq \infty, 1 \leq k \leq n$, are non-negative numbers. The case when $\left\{f_{k}\right\}$ is a non-decreasing sequence is of special interest in this case too and gives different characterizations. The corresponding result to 'Sawyer duality principle' for this discrete case was proved in [10]. This result is important for our investigations in this 
paper. Also in this discrete case it is an open question how to characterize (4) with $H$ replaced by $H_{w}$ without any further restrictions on the matrix sequence $\left\{w_{n, k}\right\}$. However, several results corresponding to (a)-(d) in the continuous case are known. So, a characterization corresponding to (d) for the case $1<p \leq q<\infty$ was proved in [11]. A characterization for the case $1<q<p<\infty$ is an open problem. However, we can consider the setting corresponding to some subclass of (d) that is as follows:

We consider the operator

$$
\left(S_{n-1} f\right)_{i}=\sum_{k_{1}=1}^{i} \omega_{1, k_{1}} \sum_{k_{2}=1}^{k_{1}} \omega_{2, k_{2}} \cdots \sum_{k_{n-1}=1}^{k_{n-2}} \omega_{n-1, k_{n-1}} \sum_{j=1}^{k_{n-1}} f_{j}, \quad i \geq 1,
$$

and call it a $n$-multiple discrete Hardy operator with weights.

Let us notice that if in (5) we change the order of summation, then the operator $S_{n-1}$ can be rewritten in the form

$$
\left(S_{n-1} f\right)_{i}=\sum_{j=1}^{i} A_{n-1,1}(i, j) f_{j}, \quad i \geq 1,
$$

where

$$
A_{n-1,1}(i, j)=\sum_{k_{n-1}=j}^{i} \omega_{n-1, k_{n-1}} \sum_{k_{n-2}=k_{n-1}}^{i} \omega_{n-2, k_{n-2}} \cdots \sum_{k_{1}=k_{2}}^{i} \omega_{1, k_{1}}, \quad i \geq j \geq 1,
$$

for $n>1$ and $A_{n-1,1}(i, j)=1$ for $n=1$.

One main problem is to find necessary and sufficient conditions on the weight sequences $\omega_{i}, i=1,2, \ldots, n-1, u$ and $v$ for the validity of the inequality

$$
\left(\sum_{i=1}^{\infty} u_{i}^{q}\left(\sum_{j=1}^{i} A_{n-1,1}(i, j) f_{j}\right)^{q}\right)^{\frac{1}{q}} \leq C\left(\sum_{i=1}^{\infty} v_{i}^{p} f_{i}^{p}\right)^{\frac{1}{p}}
$$

for non-negative non-increasing sequences $f=\left\{f_{k}\right\}_{k=1}^{\infty}$ in the case $1<q<p<\infty$.

When $n=1$ the operator $S_{n-1}$ becomes the standard discrete Hardy operator $\left(S_{0} f\right)_{i}=$ $\sum_{j=1}^{i} f_{j}$. The validity of inequality (6) with the standard discrete Hardy operator has been in detail investigated for non-negative sequences $f$ and different relations between the parameters $p$ and $q$. A thorough analysis and review of the development of this problem can be found e.g. in $[4,12]$ and [13]. For this case and when the sequence $\left\{f_{k}\right\}$ is nonincreasing we refer to the important paper [14] and the references given there.

Concerning the general inequality (6) the following is known:

$(\alpha)$ for the case when $\left\{f_{k}\right\}$ is a non-negative sequence a characterization of (6) for the case $1<p \leq q<\infty$ was derived in [15] while a corresponding characterization for the case $1<q<p<\infty$ can be found in [16];

$(\beta)$ for the case when $\left\{f_{k}\right\}$ is a non-increasing sequence a characterization of (6) for the case $1<p \leq q<\infty$ was derived in [17].

However, so far no such characterization in the case $1<q<p<\infty$ is known and it is the main aim of this paper to fill in this gap. Our main result reads as follows. 
Theorem 2.1 Let $1<q<p<\infty$ and $n \geq 1$. Suppose that $V_{k}=\sum_{i=1}^{k} v_{i}^{p}$ when $k \geq 1$ and $V_{\infty}=\lim _{k \rightarrow \infty} V_{k}$. Then inequality (6) holds for all non-negative non-increasing sequences $f=\left\{f_{i}\right\}_{i=1}^{\infty}$ from $l_{p, v}$ if and only if $E_{12}=\max \left\{E_{1}, E_{2}\right\}<\infty$ when $V_{\infty}=\infty$ and $E_{13}=$ $\max \left\{E_{1}, E_{2}^{\prime}, E_{3}\right\}<\infty$ when $V_{\infty}<\infty$, where

$$
\begin{aligned}
E_{1}:= & \max _{0 \leq r \leq n-1}\left(\operatorname { m a x } _ { 0 \leq \tau \leq r } \left(\sum_{i=1}^{\infty}\left(\sum_{j=i}^{\infty} A_{\tau, 1}^{q}(j, i) u_{j}^{q}\right)^{\frac{q}{p-q}}\right.\right. \\
& \times\left(\sum_{k=1}^{i} A_{r, \tau+1}^{p^{\prime}}(i, k)\left(\sum_{j=1}^{k} A_{n-1, r+1}(k, j)\right)^{p^{\prime}}\left(V_{k}^{-\frac{p^{\prime}}{p}}-V_{k+1}^{-\frac{p^{\prime}}{p}}\right)\right)^{\frac{q(p-1)}{p-q}} \\
& \left.\left.\times \Delta_{i}^{+}\left(\sum_{j=i}^{\infty} A_{\tau, 1}^{q}(j, i) u_{j}^{q}\right)\right)^{\frac{p-q}{p q}}\right), \\
E_{2}:= & \sum_{s=1}^{\infty}\left(V_{s}^{-\frac{p^{\prime}}{p}}-V_{s+1}^{-\frac{p^{\prime}}{p}}\right) V_{s}^{\frac{-p^{\prime}(q-1)}{p-q}}\left(\sum_{k=1}^{s}\left(\sum_{j=1}^{k} A_{n-1,1}(k, j)\right)^{q} u_{k}^{q}\right)^{\frac{p}{p-q}}, \\
E_{2}^{\prime}:= & \sum_{s=1}^{\infty}\left(V_{s}^{-\frac{p^{\prime}}{p}}-V_{s+1}^{-\frac{p^{\prime}}{p}}\right)\left(V_{s}^{-\frac{p^{\prime}}{p}}-V_{\infty}^{-\frac{p^{\prime}}{p}}\right)^{\frac{p(q-1)}{p-q}}\left(\sum_{k=1}^{s}\left(\sum_{j=1}^{k} A_{n-1,1}(k, j)\right)^{q} u_{k}^{q}\right)^{\frac{p}{p-q}},
\end{aligned}
$$

and

$$
E_{3}:=V_{\infty}^{-\frac{1}{p}}\left(\sum_{i=1}^{\infty}\left(\sum_{j=1}^{i} A_{n-1,1}(i, j)\right)^{q} u_{i}^{q}\right)^{\frac{1}{q}} .
$$

Moreover, $E_{12} \approx C$ when $V_{\infty}=\infty$ and $E_{13} \approx C$ when $V_{\infty}<\infty$, where $C$ is the best constant in (6).

\section{Notations and auxiliary statements}

Let $\frac{1}{p}+\frac{1}{p^{\prime}}=1$ and $\frac{1}{q}+\frac{1}{q^{\prime}}=1$. The symbol $A \ll B$ means that $A \leq C B$ with some constant $C$, which may depend only on the parameters $p$ and $q$. Moreover, if $A \ll B \ll A$, then we write $A \approx B$.

For all $i \geq j \geq 1$ we suppose that $A_{l, m}(i, j)=1$ when $l<m$ and $A_{l, m}(i, j)=\sum_{k_{l}=j}^{i} \omega_{l, k_{l}} \times$ $\sum_{k_{l-1}=k_{l}}^{i} \omega_{l-1, k_{l-1}} \cdots \sum_{k_{m}=k_{m+1}}^{i} \omega_{m, k_{m}}$ for $n-1 \geq l \geq m \geq 1$. Moreover, for all $i<j$ we suppose that $A_{l, m}(i, j)=0$ when $l, m \geq 1$.

In [15] the following lemma was proved.

Lemma A For all $i, j, \tau: 1 \leq j \leq \tau \leq i$ the estimates

$$
\max _{m-1 \leq r \leq l} A_{r, m}(i, \tau) A_{l, r+1}(\tau, j) \leq A_{l, m}(i, j) \leq \sum_{r=m-1}^{l} A_{r, m}(i, \tau) A_{l, r+1}(\tau, j)
$$

hold when $n-1 \geq l \geq m \geq 1$.

We also need the following discrete analog of the 'Sawyer duality principle', which was proved in [10]. 
Theorem A Let $1<p, q<\infty$. Let $\left(a_{i, j}\right)$ be a triangle matrix with $a_{i, j} \geq 0$ when $i \geq j \geq 1$ and $a_{i, j}=0$ when $i<j$. Let $V_{k}=\sum_{i=1}^{k} p_{i}^{p}$ when $k \geq 1$ and $V_{\infty}=\lim _{k \rightarrow \infty} V_{k}$. Then the inequality

$$
\left(\sum_{i=1}^{\infty} u_{i}^{q}\left(\sum_{j=1}^{i} a_{i, j} f_{j}\right)^{q}\right)^{\frac{1}{q}} \leq C\left(\sum_{i=1}^{\infty} v_{i}^{p} f_{i}^{p}\right)^{\frac{1}{p}}
$$

for all non-negative non-increasing sequences $f=\left\{f_{i}\right\}_{i=1}^{\infty}$ from $l_{p, v}$ is equivalent to the inequality

$$
\left(\sum_{k=1}^{\infty}\left(\sum_{j=1}^{k} \sum_{i=j}^{\infty} a_{i, j} g_{i}\right)^{p^{\prime}}\left(V_{k}^{-\frac{p^{\prime}}{p}}-V_{k+1}^{-\frac{p^{\prime}}{p}}\right)\right)^{\frac{1}{p^{\prime}}} \leq \widetilde{C}\left(\sum_{i=1}^{\infty} g_{i}^{q^{\prime}} u_{i}^{-q^{\prime}}\right)^{\frac{1}{q^{\prime}}}
$$

for all non-negative sequences $g=\left\{g_{i}\right\}_{i=1}^{\infty}$ when $V_{\infty}=\infty$, and it is equivalent to the inequality

$$
\begin{aligned}
& \left(\sum_{k=1}^{\infty}\left(\sum_{j=1}^{k} \sum_{i=j}^{\infty} a_{i, j} g_{i}\right)^{p^{\prime}}\left(V_{k}^{-\frac{p^{\prime}}{p}}-V_{k+1}^{-\frac{p^{\prime}}{p}}\right)\right)^{\frac{1}{p^{\prime}}} \\
& +\left(\sum_{j=1}^{\infty} \sum_{i=j}^{\infty} a_{i, j} g_{i}\right)\left(\sum_{k=1}^{\infty} v_{k}\right)^{-\frac{1}{p}} \leq \bar{C}\left(\sum_{i=1}^{\infty} g_{i}^{q^{\prime}} u_{i}^{-q^{\prime}}\right)^{\frac{1}{q^{\prime}}}
\end{aligned}
$$

for all non-negative sequences $g=\left\{g_{i}\right\}_{i=1}^{\infty}$ when $V_{\infty}<\infty$.

Moreover, $\widetilde{C} \approx C$ when $V_{\infty}=\infty$ and $\bar{C} \approx C$ when $V_{\infty}<\infty$, where $C, \widetilde{C}$ and $\bar{C}$ are the best constants in (12), (13) and (14), respectively.

We also need the following well-known result (see e.g. [4], p.58).

Theorem B Let $1<q<p<\infty$. Let $\left\{\alpha_{i}\right\}_{i=1}^{\infty}$ be a non-negative sequence. Then the inequality

$$
\left(\sum_{i=1}^{\infty} u_{i}^{q}\left(\sum_{j=1}^{i} \alpha_{j} f_{j}\right)^{q}\right)^{\frac{1}{q}} \leq C\left(\sum_{i=1}^{\infty} v_{i}^{p} f_{i}^{p}\right)^{\frac{1}{p}}
$$

holds for all non-negative sequences $f=\left\{f_{i}\right\}_{i=1}^{\infty}$ from $l_{p, v}$ if and only if

$$
H:=\sum_{i=1}^{\infty}\left(u_{i}^{q}\left(\sum_{j=i}^{\infty} u_{j}^{q}\right)^{\frac{q}{p-q}}\left(\sum_{j=1}^{i} \alpha_{j}^{p^{\prime}} v_{j}^{-p^{\prime}}\right)^{\frac{q(p-1)}{p-q}}\right)<\infty .
$$

Moreover, $H \approx C$, where $C$ is the best constant in (15).

We also need two theorems from [16].

The first theorem presents conditions for the validity of inequality (6) for only nonnegative sequences. Here we consider absolutely the same problem but with monotonicity restriction. Thus, it helps us to compare the results with and without monotonicity restriction. In addition, we need it to illustrate some applications given in the last section of the presented paper. 
The second theorem studies inequality (6) for the dual operator $S_{n-1}^{*}$ to the operator $S_{n-1}$ defined by

$$
\left(S_{n-1}^{*} f\right)_{i}=\sum_{j=i}^{\infty} A_{n-1,1}(j, i) f_{j}, \quad i \geq 1 .
$$

Theorem C Let $1<q<p<\infty$ and $n \geq 1$. Then inequality (6) holds for all non-negative sequences $f=\left\{f_{i}\right\}_{i=1}^{\infty}$ from $l_{p, v}$ if and only if

$$
\begin{aligned}
A(n):= & \max _{0 \leq \tau \leq n-1}\left(\sum_{i=1}^{\infty}\left(\sum_{j=i}^{\infty} A_{\tau, 1}^{q}(j, i) u_{j}^{q}\right)^{\frac{q}{p-q}}\left(\sum_{k=1}^{i} A_{n-1, \tau+1}^{p^{\prime}}(i, k) v_{k}^{-p^{\prime}}\right)^{\frac{q(p-1)}{p-q}}\right. \\
& \left.\times \Delta_{i}^{+}\left(\sum_{j=i}^{\infty} A_{\tau, 1}^{q}(j, i) u_{j}^{q}\right)\right)^{\frac{p-q}{p q}}<\infty
\end{aligned}
$$

where $\Delta_{i}^{+} E_{j, i}=E_{j, i}-E_{j, i+1}$.

Moreover, $A(n) \approx C$, where $C$ is the best constant in (6).

Theorem D Let $1<q<p<\infty$ and $n \geq 1$. Then the inequality

$$
\left(\sum_{i=1}^{\infty} u_{i}^{q}\left(\sum_{j=i}^{\infty} A_{n-1,1}(j, i) f_{j}\right)^{q}\right)^{\frac{1}{q}} \leq C\left(\sum_{i=1}^{\infty} v_{i}^{p} f_{i}^{p}\right)^{\frac{1}{p}}
$$

holds for all non-negative sequences $f=\left\{f_{i}\right\}_{i=1}^{\infty}$ from $l_{p, v}$ if and only if

$$
\begin{aligned}
B(n):= & \max _{0 \leq \tau \leq n-1}\left(\sum_{i=1}^{\infty}\left(\sum_{j=i}^{\infty} A_{\tau, 1}^{p^{\prime}}(j, i) v_{j}^{-p^{\prime}}\right)^{\frac{p(q-1)}{p-q}}\left(\sum_{k=1}^{i} A_{n-1, \tau+1}^{q}(i, k) u_{k}^{q}\right)^{\frac{p}{p-q}}\right. \\
& \left.\times \Delta_{i}^{+}\left(\sum_{j=i}^{\infty} A_{\tau, 1}^{p^{\prime}}(j, i) v_{j}^{-p^{\prime}}\right)\right)^{\frac{p-q}{p q}}<\infty .
\end{aligned}
$$

Moreover, $B(n) \approx C$, where $C$ is the best constant in (17).

\section{Proof of Theorem 2.1}

We start our proof from the case $V_{\infty}=\infty$.

Assuming $a_{i, j} \equiv A_{n-1,1}(i, j)$, by Theorem A we see that inequality (6) for all non-negative non-increasing sequences $f=\left\{f_{i}\right\}_{i=1}^{\infty}$ from $l_{p, v}$ is equivalent to the inequality

$$
\left(\sum_{k=1}^{\infty}\left(\sum_{j=1}^{k} \sum_{i=j}^{\infty} A_{n-1,1}(i, j) g_{i}\right)^{p^{\prime}}\left(V_{k}^{-\frac{p^{\prime}}{p}}-V_{k+1}^{-\frac{p^{\prime}}{p}}\right)\right)^{\frac{1}{p^{\prime}}} \leq \widetilde{C}\left(\sum_{i=1}^{\infty} g_{i}^{q^{\prime}} u_{i}^{-q^{\prime}}\right)^{\frac{1}{q^{\prime}}}
$$

for all non-negative sequences $g=\left\{g_{i}\right\}_{i=1}^{\infty}$.

Denote

$$
(\widetilde{S} f)_{k}=\sum_{j=1}^{k} \sum_{i=j}^{\infty} A_{n-1,1}(i, j) g_{i}
$$


It is obvious that

$$
(\widetilde{S} f)_{k} \approx \sum_{j=1}^{k} \sum_{i=j}^{k} A_{n-1,1}(i, j) g_{i}+\sum_{j=1}^{k} \sum_{i=k}^{\infty} A_{n-1,1}(i, j) g_{i} .
$$

Since $A_{n-1,1}(i, j)$ and $g_{i}$ are non-negative, we can change the order of summation in both terms:

$$
(\widetilde{S} f)_{k} \approx \sum_{i=1}^{k} \sum_{j=1}^{i} A_{n-1,1}(i, j) g_{i}+\sum_{i=k}^{\infty} \sum_{j=1}^{k} A_{n-1,1}(i, j) g_{i} .
$$

Let us, respectively, denote

$$
\left(\widetilde{S}_{1} f\right)_{k}=\sum_{i=1}^{k} \sum_{j=1}^{i} A_{n-1,1}(i, j) g_{i}=\sum_{i=1}^{k} \widetilde{A}_{i} g_{i}
$$

where $\widetilde{A}_{i}=\sum_{j=1}^{i} A_{n-1,1}(i, j)$, and

$$
\left(\widetilde{S}_{2} f\right)_{k}=\sum_{i=k}^{\infty} \sum_{j=1}^{k} A_{n-1,1}(i, j) g_{i}=\sum_{i=k}^{\infty} \widetilde{A}_{n-1,1}(i, k) g_{i}
$$

where $\widetilde{A}_{n-1,1}(i, k)=\sum_{j=1}^{k} A_{n-1,1}(i, j)$.

Next, we will work with $\widetilde{A}_{n-1,1}(i, k)$. Since $i \geq k \geq j$, then from (11) we see that

$$
A_{n-1,1}(i, j) \approx \sum_{r=0}^{n-1} A_{r, 1}(i, k) A_{n-1, r+1}(k, j)
$$

Substitute it in $\widetilde{A}_{n-1,1}(i, k)$ and we find that

$$
\begin{aligned}
\widetilde{A}_{n-1,1}(i, k) & \approx \sum_{j=1}^{k} \sum_{r=0}^{n-1} A_{r, 1}(i, k) A_{n-1, r+1}(k, j) \\
& =\sum_{r=0}^{n-1} A_{r, 1}(i, k) \sum_{j=1}^{k} A_{n-1, r+1}(k, j)=\sum_{r=0}^{n-1} A_{r, 1}(i, k) \cdot b_{k, r},
\end{aligned}
$$

where $b_{k, r}=\sum_{j=1}^{k} A_{n-1, r+1}(k, j)$.

Therefore, the validity of inequality (18) is equivalent to the simultaneous validity of the following inequalities:

$$
\left(\sum_{k=1}^{\infty}\left(\sum_{i=1}^{k} \widetilde{A}_{i} g_{i}\right)^{p^{\prime}}\left(V_{k}^{-\frac{p^{\prime}}{p}}-V_{k+1}^{-\frac{p^{\prime}}{p}}\right)\right)^{\frac{1}{p^{\prime}}} \leq \widetilde{C}_{1}\left(\sum_{i=1}^{\infty} u_{i}^{-q^{\prime}} g_{i}^{q^{\prime}}\right)^{\frac{1}{q^{\prime}}}
$$

and

$$
\left(\sum_{k=1}^{\infty}\left(\sum_{i=k}^{\infty} A_{r, 1}(i, k) g_{i}\right)^{p^{\prime}} b_{k, r}^{p^{\prime}}\left(V_{k}^{-\frac{p^{\prime}}{p}}-V_{k+1}^{-\frac{p^{\prime}}{p}}\right)\right)^{\frac{1}{p^{\prime}}} \leq \widetilde{C}_{2, r}\left(\sum_{i=1}^{\infty} u_{i}^{-q^{\prime}} g_{i}^{q^{\prime}}\right)^{\frac{1}{q^{\prime}}}
$$


where $0 \leq r \leq n-1$. Moreover, if $\widetilde{C}_{2}=\max _{0 \leq r \leq n-1} \widetilde{C}_{2, r}$, then

$$
\widetilde{C} \approx \max \left\{\widetilde{C}_{1}, \widetilde{C}_{2}\right\}
$$

It is obvious that inequality (19) is a standard Hardy inequality. Hence, according to Theorem B, inequality (19) holds if and only if $E_{2}<\infty$. Moreover, we take into account the following fact:

$$
\sum_{j=s}^{\infty}\left(V_{j}^{-\frac{p^{\prime}}{p}}-V_{j+1}^{-\frac{p^{\prime}}{p}}\right)=V_{s}^{-\frac{p^{\prime}}{p}}
$$

There are $n$ inequalities in (20). All of these $n$ inequalities can be characterized by Theorem $\mathrm{D}$. It means that the condition $E_{1}<\infty$ is necessary and sufficient for the validity of (20). Moreover, in view of Theorem $B$, Theorem $D$ and (21), we see that $E_{12}=\max \left\{E_{1}, E_{2}\right\} \approx$ $C$, where $C$ is the best constant in (6).

Let us turn to the case $V_{\infty}<\infty$. The difference from the previous situation is that by Theorem A inequality (6) holds if and only if (18) holds with some other constant $\bar{C}_{1}$ instead of $\widetilde{C}$, and

$$
\left(\sum_{j=1}^{\infty} \sum_{i=j}^{\infty} A_{n-1,1}(i, j) g_{i}\right)\left(\sum_{k=1}^{\infty} v_{k}\right)^{-\frac{1}{p}} \leq \bar{C}_{2}\left(\sum_{i=1}^{\infty} g_{i}^{q^{\prime}} u_{i}^{-q^{\prime}}\right)^{\frac{1}{q^{\prime}}}
$$

simultaneously holds for all non-negative sequences $g=\left\{g_{i}\right\}_{i=1}^{\infty}$. Moreover,

$$
\bar{C} \approx \max \left\{\bar{C}_{1}, \bar{C}_{2}\right\}
$$

By arguing as in the previous case, we see that inequality (18) holds if and only if the conditions $E_{1}<\infty$ and $E_{2}^{\prime}<\infty$ hold. Let us notice that the difference of the expressions $E_{2}$ and $E_{2}^{\prime}$ is only in the fact that we need to take into account the relation

$$
\sum_{j=s}^{\infty}\left(V_{j}^{-\frac{p^{\prime}}{p}}-V_{j+1}^{-\frac{p^{\prime}}{p}}\right)=V_{s}^{-\frac{p^{\prime}}{p}}-V_{\infty}^{-\frac{p^{\prime}}{p}}
$$

instead of the relation (22).

Next, we study inequality (23). Since $A_{n-1,1}(i, j)$ and $g_{i}$ are non-negative, we can change the order of summation in the first bracket of (23) and obtain

$$
\sum_{i=1}^{\infty} \sum_{j=1}^{i} A_{n-1,1}(i, j) g_{i} \leq \bar{C}_{2} V_{\infty}^{\frac{1}{p}}\left(\sum_{i=1}^{\infty} g_{i}^{q^{\prime}} u_{i}^{-q^{\prime}}\right)^{\frac{1}{q^{\prime}}}
$$

If we apply the reverse Hölder inequality to the last expression, we get

$$
\left(\sum_{i=1}^{\infty}\left(\sum_{j=1}^{i} A_{n-1,1}(i, j)\right)^{q} u_{i}^{q}\right)^{\frac{1}{q}}=\bar{C}_{2} V_{\infty}^{\frac{1}{p}}
$$


Therefore, inequality (23) holds if and only if $E_{3}<\infty$. Moreover, by Theorem B, Theorem $\mathrm{D}$ and (24), we see that $E_{13}=\max \left\{E_{1}, E_{2}^{\prime}, E_{3}\right\} \approx C$, where $C$ is the best constant in (6). The proof of Theorem 2.1 is complete.

\section{Applications}

In the theory of series the estimates of norms of summable matrices are very important problems. One of the important methods of summation is Hölder's method by $(H, n-1)$ defined as follows:

The series $\sum_{j=1}^{\infty} f_{j}$ is summable by Hölder's $(H, n-1)$ method with the sum $S$ if $\lim _{i \rightarrow \infty}\left(H_{n-1} f\right)_{i}=S$, where

$$
\begin{aligned}
& \left(H_{0} f\right)_{i}=f_{1}+f_{2}+\cdots+f_{i}, \\
& \left(H_{n-1} f\right)_{i}=\frac{\left(H_{n-2} f\right)_{1}+\left(H_{n-2} f\right)_{2}+\cdots+\left(H_{n-2} f\right)_{i}}{i}, \quad i=1,2, \ldots
\end{aligned}
$$

This method, introduced in 1882 by Hölder in [9], is a generalization of the summation method of arithmetic averages. It is obvious that $(H, 1)$ is the method of arithmetic averages in the ordinary sense.

In [17] it was shown that $H_{n-1}$ can be presented in the following form:

$$
\left(H_{n-1} f\right)_{i}=\frac{1}{i} \sum_{k_{1}=1}^{i} \frac{1}{k_{1}} \sum_{k_{2}=1}^{k_{1}} \frac{1}{k_{2}} \ldots \sum_{k_{n-2}=1}^{k_{n-3}} \frac{1}{k_{n-2}} \sum_{k_{n-1}=1}^{k_{n-2}} \sum_{j=1}^{k_{n-1}} f_{j} .
$$

If $w_{m, k_{m}} \equiv \frac{1}{k_{m}}$ for $1 \leq m \leq n-2$ and $w_{n-1, k_{n-1}} \equiv 1$, then we see that the operator $H_{n-1}$ is a particular case of our operator $S_{n-1}$, namely:

$$
\left(S_{n-1} f\right)_{i}=i\left(H_{n-1} f\right)_{i}
$$

If we change the order of summation, then we find that for this particular operator $S_{n-1}$ the summation matrix $A_{n-1,1}$ has the following form:

$$
A_{n-1,1}(i, j)=\sum_{k_{n-1}=j}^{i} \sum_{k_{n-2}=k_{n-1}}^{i} \frac{1}{k_{n-2}} \cdots \sum_{k_{2}=k_{3}}^{i} \frac{1}{k_{2}} \sum_{k_{1}=k_{2}}^{i} \frac{1}{k_{1}}, \quad i \geq j \geq 1 .
$$

Therefore, we can consider the following inequality:

$$
\left(\sum_{i=1}^{\infty} \widetilde{u}_{i}^{q}\left(H_{n-1} f\right)_{i}^{q}\right)^{\frac{1}{q}} \leq C\left(\sum_{i=1}^{\infty} v_{i}^{p} f_{i}^{p}\right)^{\frac{1}{p}}
$$

which is equivalent to inequality (6), where, in view of (25), $u_{i}=\frac{\widetilde{u}_{i}}{i}$. Thus, according to Theorem $\mathrm{C}$ and Theorem 2.1 we have the following results.

Theorem 5.1 Let $1<q<p<\infty$ and $n \geq 1$. Then inequality (27) holds for all non-negative sequences $f=\left\{f_{i}\right\}_{i=1}^{\infty}$ from $l_{p, v}$ if and only if $A(n)<\infty$ (see (16)), where $A_{n-1,1}$ is defined in (26) and $u_{i}=\frac{\widetilde{u}_{i}}{i}$. Moreover, $A(n) \approx C$, where $C$ is the best constant in (27). 
Theorem 5.2 Let $1<q<p<\infty$ and $n \geq 1$. Suppose that $V_{k}=\sum_{i=1}^{k} v_{i}^{p}$ when $k \geq 1$ and $V_{\infty}=\lim _{k \rightarrow \infty} V_{k}$. Then inequality (27) holds for all non-negative non-increasing sequences $f=\left\{f_{i}\right\}_{i=1}^{\infty}$ from $l_{p, v}$ if and only if $E_{12}=\max \left\{E_{1}, E_{2}\right\}<\infty$ when $V_{\infty}=\infty$ (see (7) and (8)) and $E_{13}=\max \left\{E_{1}, E_{2}^{\prime}, E_{3}\right\}<\infty$ when $V_{\infty}<\infty$ (see (7), (9), and (10)), where $A_{n-1,1}$ is defined in (26) and $u_{i}=\frac{\widetilde{u}_{i}}{i}$. Moreover, $E_{12} \approx C$ when $V_{\infty}=\infty$ and $E_{13} \approx C$ when $V_{\infty}<\infty$, where $C$ is the best constant in (27).

\section{Competing interests}

The authors declare that they have no competing interests.

\section{Authors' contributions}

All three authors have on an equal level discussed and posed the research questions in this paper. AK has substantially helped to prove the main results and to type the manuscript. AT is the main author concerning the proofs of the main results and typing of the manuscript. LEP has put the results into a more general frame in the introduction and instructed how to write the paper in this final form. All authors read and approved the final manuscript.

\section{Author details}

${ }^{1}$ KIMEP University, Abai Ave. 4, Almaty, 050010, Kazakhstan. ${ }^{2}$ Luleå University of Technology, Luleå, 971 87, Sweden. ${ }^{3}$ Narvik University College, P.O. Box 385, Narvik, 8505, Norway. ${ }^{4}$ L.N. Gumilyov Eurasian National University, Munaytpasov St. 5, Astana, 010008, Kazakhstan.

\section{Acknowledgements}

The authors would like to thank Professor Ryskul Oinarov for his generous suggestions which have improved this paper. The paper was written under financial support by the Scientific Committee of the Ministry of Education and Science of Kazakhstan, Grant No. 5495/GF4 on priority area 'Intellectual potential of the country'.

Received: 16 April 2015 Accepted: 25 September 2015 Published online: 06 October 2015

\section{References}

1. Hardy, GH: Notes on a theorem of Hilbert. Math. Z. 6, 314-317 (1920)

2. Hardy, GH: Notes on some points in the integral calculus, LX. An inequality between integrals. Messenger Math. 54 , 150-156 (1925)

3. Kufner, A, Persson, L-E: Weighted Inequalities of Hardy Type. World Scientific, Singapore (2003)

4. Kufner, A, Maligranda, L, Persson, L-E: The Hardy Inequality: About Its History and Some Related Results. Vydavatelský Servis, Pilsen (2007)

5. Kokilashvili, V, Meskhi, A, Persson, L-E: Weighted Norm Inequalities for Integral Transforms with Product Weights. Nova Science Publishers, New York (2010)

6. Oinarov, R: Boundedness and compactness of Volterra type integral operators. Sib. Math. J. 48, 884-896 (2007)

7. Oinarov, R: Boundedness and compactness in weighted Lebesgue spaces of integral operators with variable integration limits. Sib. Math. J. 52, 1042-1055 (2011)

8. Sawyer, E: Boundedness of classical operators on classical Lorentz spaces. Stud. Math. 2, 145-158 (1990)

9. Hölder, O: Grenzwerthe von Reihen an der Konvergenzgrenze. Math. Ann. 20, 535-549 (1882)

10. Oinarov, R, Shalgynbayeva, S: Weighted Hardy inequalities on the cone of monotone sequences. Izv. Nats. Akad. Nauk Resp. Kaz. Ser. Fiz.-Mat. 1, 33-42 (1998) (in Russian)

11. Oinarov, R, Taspaganbetova, Z: Criteria of boundedness and compactness of a class of matrix operators. J. Inequal. Appl. (2012). doi:10.1186/1029-242X-2012-53

12. Okpoti, CA: Weight characterizations of Hardy and Carleman type inequalities. Ph.D. thesis, Luleå University of Technology, Luleå, Sweden (2006)

13. Popova, O: Weighted Hardy-type inequalities on the cones of monotone and quasi-concave functions. Ph.D. thesis, Luleå University of Technology, Luleå, Sweden and Peoples' Friendship University of Russia, Moscow, Russia (2012)

14. Bennett, G, Grosse-Erdmann, K-G: Weighted Hardy inequalities for decreasing sequences and functions. Math. Ann. 3, 489-531 (2006)

15. Oinarov, R, Temirkhanova, A: Boundedness and compactness of a class of matrix operators in weighted sequence spaces. J. Math. Inequal. 2, 555-570 (2008)

16. Kalybay, A, Oinarov, R, Temirkhanova, A: Boundedness of $n$-multiple discrete Hardy operators with weights for $1<q<p<\infty$. J. Funct. Spaces Appl. (2013). doi:10.1155/2013/121767

17. Taspaganbetova, Z: Two-sided estimates for matrix operators on the cone of monotone sequences. J. Math. Anal. Appl. 410(1), 82-93 (2014) 